\title{
Students' mental construction in cube and cuboid concepts based on mathematical ability differences
}

\author{
Imam Rofiki' ${ }^{1}$ Ahmad Choirul Anam ${ }^{2}$, Putri Eka Sari' ${ }^{3}$, Wahyu Henky \\ Irawan $^{1}$, Ika Santia ${ }^{4}$ \\ ${ }^{1}$ Universitas Islam Negeri Maulana Malik Ibrahim Malang, Indonesia \\ ${ }^{2}$ Universitas Negeri Surabaya, Indonesia \\ ${ }^{3}$ Universitas Islam Negeri Sunan Ampel Surabaya, Indonesia \\ ${ }^{4}$ Universitas Nusantara PGRI Kediri, Indonesia \\ $\triangle$ imam.rofiki@uin-malang.ac.id
}

\section{Article Information \\ Submitted May 17, 2020 \\ Revised May 20, 2020 \\ Accepted June 17, 2020}

\section{Keywords}

APOS Theory; Mathematical Ability; Mental Construction ; Understanding Concepts.

\begin{abstract}
Understanding the cube and cuboid concepts is one of the essential goals of solid geometry learning. Most studies on students' understanding of these concepts have posited a gap between the conception of the surface area and volume. This research was aimed to describe the students' mental constructions in APOS (Action, Process, Object, and Schema) theory. Three junior high school students who have differences in mathematical abilities were chosen as the research subjects. This research employed the qualitative approach. The data were collected using the task sheet and interview. The result of this research discovered that the subject with high mathematical ability could solve cube and cuboid problems correctly in the action stage. In the process and object stage, the subject could provide detailed explanations of the steps to assess the surface area and volume by comparing two shapes with different sizes. In the schema stage, the subject made a systematic understanding related to the concepts of surface area and volume of cube and cuboid. The subject with medium mathematical ability explained the mental construction in action and process stage well despite the error done in the process stage. In the next stage, she compared two different shapes of cube and cuboid to find their ratio. In the last stage, the subject could not complete the explanation. The subject with low mathematical ability could solve the cube and cuboid problems. At the process, object, and schema stage, the subject could not complete the indicators of cube and cuboid concepts.
\end{abstract}

\section{INTRODUCTION}

Understanding is a cognitive process in connecting new knowledge and prior knowledge (Haylock \& Cockburn, 2008). Understanding is a crucial ability that students must have in creating cognitive relationships during the learning process (Rofiki et al., 2017). The learning process is an attempt to create conditions that involve a person's physical and mental understanding. This is by one of the mathematics learning objects listed in the 2013 curriculum, which is an understanding of mathematical concepts, explaining the relevance of concepts and applying concepts or algorithms flexibly, accurately, efficiently, and precisely to solve problems. Understanding mathematical concepts mean making connections between mental representations owned by a person and the mathematical concepts being studied (Barmby et al., 2007). Mathematics is one of the sciences that study concepts. The concept formed through a single and complex schematically structured ideas could complete the concept mastery understanding.

Published by Mathematics Education Department, UIN Raden Intan Lampung.
} 
In mathematics, it takes maximum mastery to understand a concept (Barody et al., 2007). The development of students' understanding of a concept can differ depending on how students explore mental constructs that they have specifically. Mental construction can be seen through identifying the construction of knowledge using the APOS framework (Action, Process, Object, and Schema) (Arnon et al., 2014; García \& Parraguez, 2017; Figueroa et al., 2018). One mathematical concept that can be analyzed using the APOS framework is the concept of geometry. Geometry is one aspect of mathematics learning that students must master (Anwar \& Rofiki, 2018; Puloo, 2018). Building space is one of the sub-subjects studied by students in geometry which is divided into two types, namely flat solid figure side and a curve solid figure side. Cube and cuboid are three-dimensional shapes that are often encountered in daily life, so students are expected to master the concepts of building them. Some research results indicate that students' understanding of geometry is weak (Suryani et al., 2020) and requires mental habituation to improve their understanding through learning (Kurniawan et al., 2019). Because in understanding a mathematical concept or idea that is arranged hierarchically, it takes a learning process through gradual and sequential habituation and based on previous learning experiences. Geometric concepts being studied can improve their understanding if they can process the concepts gradually. Thus, students can represent each concept according to the level of knowledge and make it a mathematical knowledge structure.

Mental activity always develops at each stage by explaining each of the hierarchical mental constructions to obtain knowledge (Bansilal et al., 2017; Mena-Lorca \& Parraguez, 2016). Dubinsky (2002) adopted Piaget's theory of reflective abstraction. Each individual tends to respond and understand mathematical concepts through the reconstruction of actions, processes, objects, and schemas in understanding and solving mathematical problems. Altieri and Schirmer (2019) reveal that the mental construction of constructed conceptions in the symbolic and embodied world of Eigen theory was based on the APOS scale by utilizing the test instrument as the learning depth measurement.

Priss (2018) argues that APOS is an understanding that uses physical and mental activity that changes objects obtained from interactions with others or results from mental construction with pre-existing objects related to mathematical ideas. The mental construction is carried out through several stages: actions, processes, objects, and schemas (Arnon et al., 2014; Dubinsky $\&$ Wilson, 2001). Action is the cognitive process when someone responds to a task externally, and then it needs specific guidance to complete the task explicitly. In other words, the solution is only carried out procedurally. Stages of the process occur when someone can predict and understand the steps in completing a task because the process entirely occurs in mind (internal). It means that someone can be said to have already done the stages of the process if he can complete a task without performing procedural steps. Object means the stage of understanding mathematical concepts that are built from the application of activities at the stage of action and process. Students can understand mathematical concepts with the help of external stimulation and then transformed them into controlled internal activities so that they can understand the meaning of these concepts (Martínez-Planel \& Delgado, 2016). The last stage is the schema which is a coherent system of action, process, and object. Students can link the activities of action, process, and object and can create other schemas. Students who carry out the schema stage can understand the concepts and relate them to concepts in other topics. 
Many studies concern in mental construction. Gray et al. (1999) investigated how students manage the transition to advanced mathematical thinking in various ways leading to a divergent continuum of attainment. Brijlall and Ndlovu (2013) reported that students may have the correct answer but lacked conceptual understanding. Ndlovu and Brijlall (2016) explored the nature of mental constructions made by pre-service teachers in the determinant concept. Their study showed that many students could convey procedures efficiently, even if the meaning of the concept is not constructed. It indicates that students mainly possess procedural knowledge of the determinant concept. García-Martínez and Parraguez (2017) used APOS theory as the framework to measure a cognitive model of the principle of mathematical induction in higher education. Ndlovu and Brijlall (2019) found that mental constructions occur within the ActionProcess-Object-Schema (APOS) theory. Oktaç (2019) examined mental constructions in linear algebra. The majority of those studies focus on university students, yet, there have been limited studies in secondary school students. Therefore, the present research focuses on students' mental construction in cube and cuboid concepts using the APOS framework. Cube and cuboid are vital concepts because they are widely used in everyday life.

Mental construction in the APOS framework shows that there were differences in each individual in describing each stage. This can be influenced by the way of thinking and ability of each individual. The novelty of this research lies in the students' mental constructions through detailed explanations at each stage in the APOS framework on cube and cuboid concepts. It differs from previous studies that use genetic composition in general about mental and mechanical construction, but lack in-depth. Hence, this study describes students' mental construction of cube and cuboid concepts based on differences in mathematical ability. The result of this research contributes to guidance for educators in improving students' understanding.

\section{METHODS}

This study employed a qualitative approach to describe students' mental construction. This research reported students' mental construction through the identification of the APOS framework in the cube and cuboid concepts in a systematic, practical, and comprehensive manners. The research subjects were junior high school students in Surabaya, starting from determining the group of students to giving a test. 37 students were chosen. Next, the student was given a test to find out the differences in mathematical abilities. The test results found that three students each had high, medium, and low mathematical abilities.

Students assigned to be the research subjects were given tasks on cube and cuboid through interviews to describe their mental construction. The instrument used in this study was a mathematics proficiency test containing modification questions from national exam questions. 8 test questions were adapted from multiple choice questions and then adjusted into description questions given to 37 students. Problem number 1 contained the numerical fraction, the material found in problem number 2; then the algebraic aspect was contained in problem number 3, and question number 4 related to linear equations of one variable. Questions numbers 5 and 6 consisted of comparison material and the last two questions were questions that contained plane figure material. Subsequently, the test results were analyzed to differentiate each student's mathematical abilities. Students who got the score more than or equal to 80 were included in groups of students with high mathematical abilities. If students got a score between 55 and 80 , 
they were included in groups of students with moderate mathematical abilities. However, if students' scores were less than 55, they were included in groups of students with low mathematical abilities. Furthermore, the three students with different mathematical abilities were given cube and cuboid tasks which aimed to identify and provide a description of mental construction. The task contained 3 items. Item 1 was used to identify and analyze the subject's understanding at the action stage. Item 2 was used to find out and analyze the subject's understanding of the action and process stage whereas item 3 was used to identify and analyze the subject's understanding of the object and schema stage. Item 3 had more complex criteria than other items because it contained applicative questions and relates to other concepts. These items were used to find out and analyze the overall understanding of the subject. Task-based interviews were conducted simultaneously to add more data.

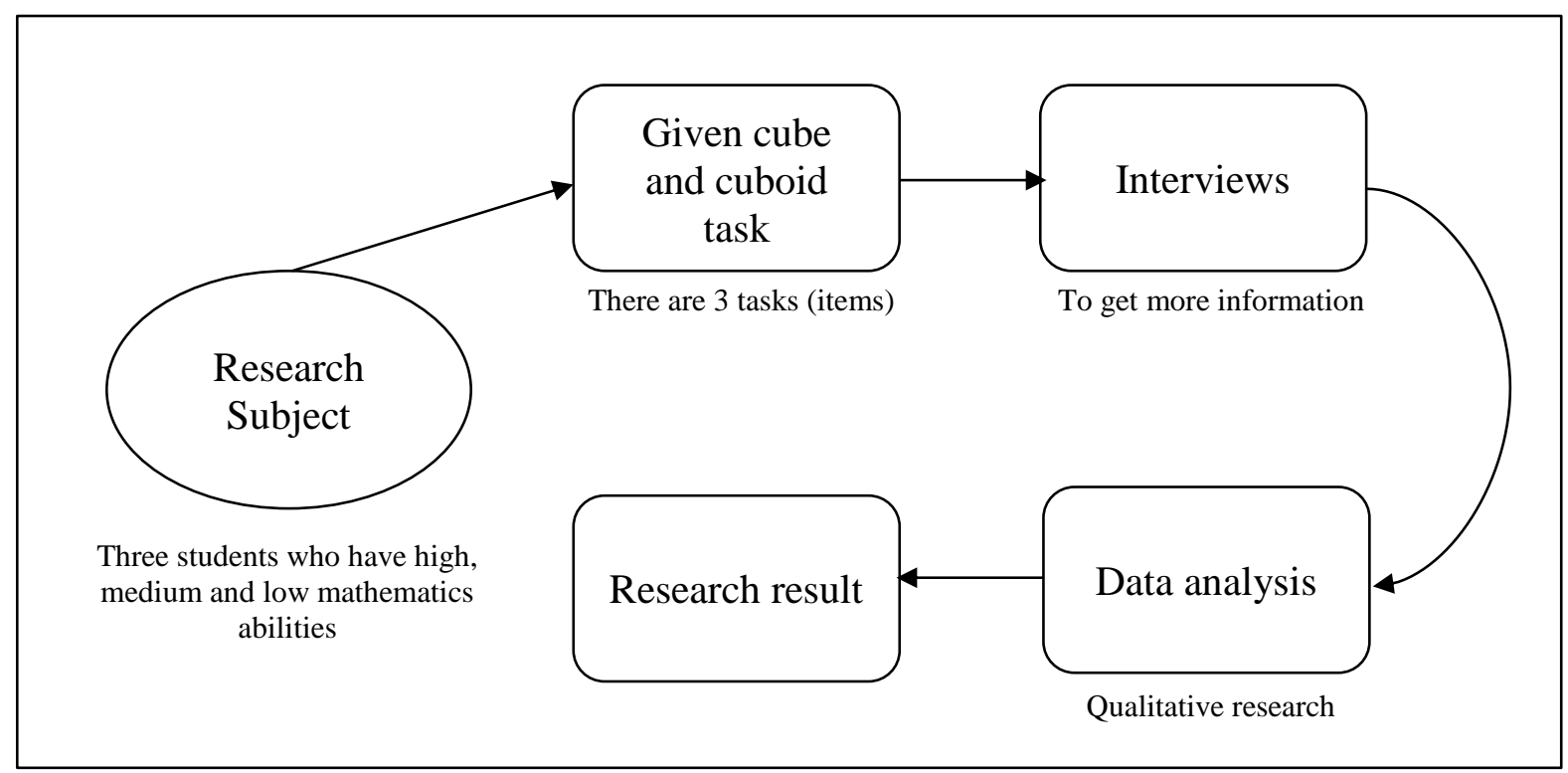

Figure 1. The Scheme of Research Method

\section{RESULTS AND DISCUSSION}

This research used a mathematics ability test and cube and cuboid task. The results of the mathematics ability test showed that 37 students were taking the test (30 females and 7 males) with the age range of 12-14. The difference in mathematical ability was known that 19 students had medium mathematical abilities. Students with low mathematical abilities were less than students with high mathematical abilities while students with high mathematical abilities were the least of the others. 


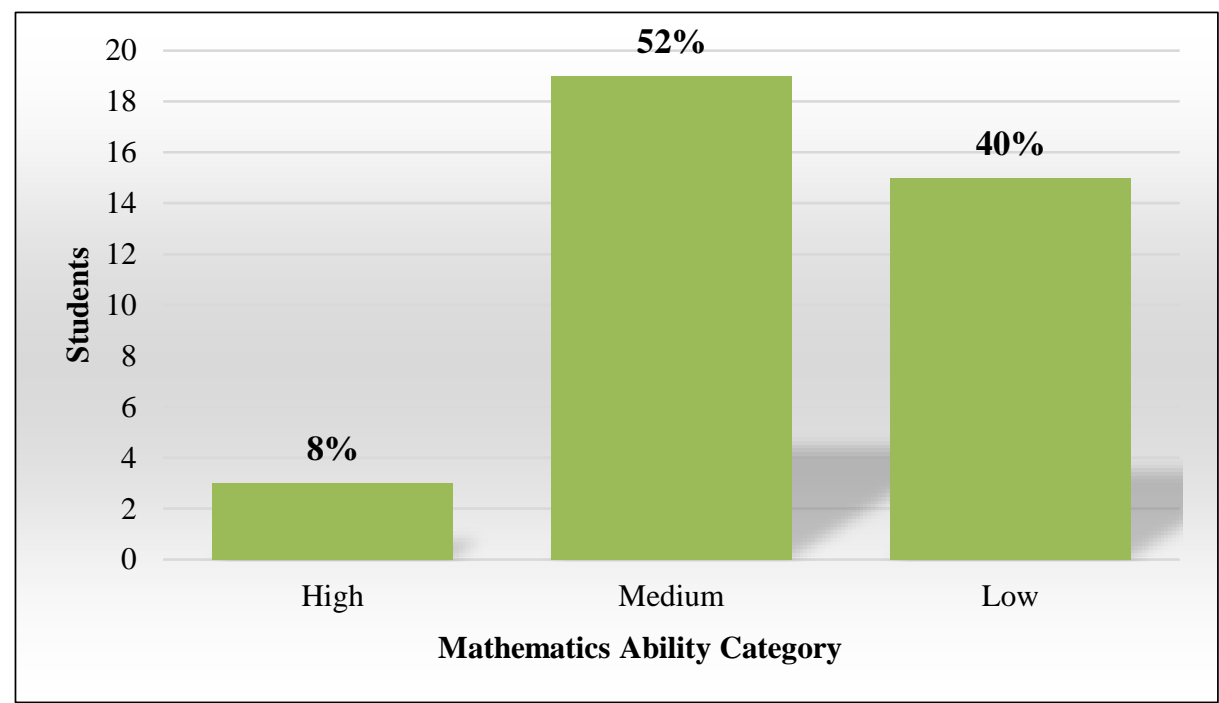

Figure 2. Students' Mathematics Ability Test Results

Based on the results of data analysis, the researchers determined and selected three students of the same sex and different mathematical abilities, each of which had high, medium, and low mathematical abilities. Detailed data from the results of tests on research subjects can be seen in Table 1.

Table 1. Classification of Mathematics Ability Test Results

\begin{tabular}{cccc}
\hline Subject & Sex & Age & Mathematical Ability \\
\hline AR & Female & 12 & High \\
KSLG & Female & 13 & Medium \\
SSAP & Female & 12 & Low \\
\hline
\end{tabular}

The subject with the initials AR was 12 years old and had a high mathematical ability. The subject with the initials KSLG was 13 years old and had a medium mathematical ability. The last subject with the initials SSAP was 12 years old and had a low mathematical ability.

The following is the description of students' mental construction using the APOS framework through cube and cuboid tasks given to research subjects. To facilitate the discussion, another term was used, namely, AR referred to as subject 1, KSLG referred to as subject 2, and SSAP referred to as subject 3 .

\subsection{The Research Result Based on Subject 1}

The cube and cuboid task was given to subject 1 . She could solve all the problems of the task well proven from the results of tests and interviews related to the concept of the cube and cuboid through the APOS framework.

\subsubsection{Action Stage}

At the action stage, the subject could understand the problem and re-explained the purpose of the problem. She also solved the problem by determining the surface area and volume of the cubes and cuboids. The figure elements used to determine the surface area of cubes and cuboids could be explained correctly, as well as the elements for determining the volume. Someone who constructs his understanding at the action stage has completed a correct procedural task (Dubinsky, 2002). Thus, subject 1 had performed the action stage. 


\subsubsection{Process Stage}

Subject 1 could understand the problem in item number 2 that corresponded to the process step indicator. At this stage, the subject was expected to understand the concepts related to cube and cuboid by showing steps in determining surface area and volume. The subject could re-explain the purpose of the problem in the questions as shown in the following interview transcript.

Interviewer : How did you understand item number 2?

Subject $1 \quad$ : Look, sir, I was asked to pay attention to the picture of the cube and cuboid. Then, I was asked to explain the decisive steps surface area and volume of cubes and cuboids.

Interviewer: Alright, try to explain the steps?

Subject 1 : first, I found the length of the unknown side. To find the surface area, I divided the cuboids into two figures of cubes because, in the problem, it was known that the length of the cuboid equals twice the cube. Of the three cubes, there was a side area that did not be counted because it was squeezed by another figure. I supposed K.1 (first cube) had 5 sides because the other side was closed up, K.2 (Second cube) had 4 sides because the other sides were squeezed by other figures, and the last, K.3 (third cube) had 5 sides because the other side was closed up. To determine the volume, I calculated the volume of the cube then multiply it by 3 because there were three cubes.

Interviewer : What was the surface area and volume?

Subject 1 : To determine the surface area of the cube, I used those steps earlier, sir:

$$
\begin{aligned}
L p=(5+ & 5+4) \times 9^{2} \\
& =(5+5+4) \times 9 \times 9 \\
& =14 \times 9 \times 9 \\
& =126 \times 9=1134 \mathrm{~cm}^{2}
\end{aligned}
$$

I see, sir. Next, I calculated the volume of the cube with the following formula. Volume $=s^{3}=9^{3}=9 \times 9 \times 9=729 \mathrm{~cm}^{3}$, then I multiplied it by 3 .

$$
\text { So, } 729 \times 3=2187 \mathrm{~cm}^{3} \text {. }
$$

Subject 1 explained the purpose of the problem and showed the steps to solve it based on the interview transcript. Subject 1 used an alternative settlement by dividing the cuboid into two equal parts because it was known that the cuboid's length was twice the length of the cube. She used the alternative because the solution was much easier. After all, the focus of the work was only on the cube. Figure 2 shows the results of subject 1's work in item number 2.

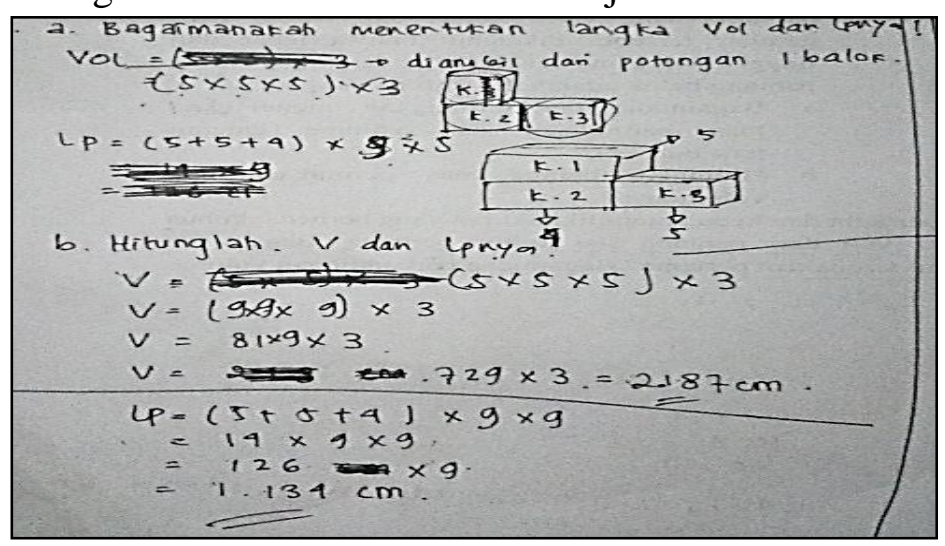

Figure 2. Subject 1 Work Results in Item Number 2 
Figure 2 shows that subject 1 answered correctly and could explain the steps. Thus, subject 1 had fulfilled the criteria of the indicator in the process stage well.

\subsubsection{Object Stage}

This stage was aimed to determine the subject's understanding of the comparison of two cubes or cuboids if their size changes. The item was suited to the objective of the object stage. Subject 1 could understand the problem well as evidenced by the explanation in answering the interview questions. She showed the comparative purpose of two shapes if the size changes twice by making an example if a cube had a side length of $10 \mathrm{~cm}$, then the change figure will have a side length of $10 \times 2=20 \mathrm{~cm}$. The following figure is the results of subject 1's work in item number 3 .

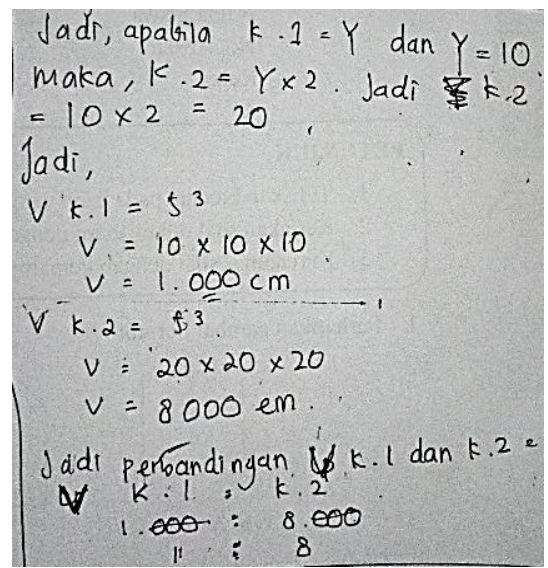

Figure 3. Subjects 1 Work Results in Item Number 3

Figure 3 shows that subject 1 made an example by displaying the side length of a $10 \mathrm{~cm}$ cube. Then, if another cube changed twice, the length would become $20 \mathrm{~cm}$. Subject 1 calculated the volume by Figure 3 and explained the stages during the interview that the length of the side changes twice, so the volume of the figure should not change twice, but 1:8. That was evidenced by the calculation of the volume of a cube with a length of $10 \mathrm{~cm}$, so the volume was $1000 \mathrm{~cm}^{3}$. If the length of the side changed twice to $20 \mathrm{~cm}$, the volume should be $8000 \mathrm{~cm}^{3}$. Thus, subject 1 could understand the comparison of a cube correctly and adequately.

\subsubsection{Schema Stage}

The merging of the action, process, and object formed a mutually coherent schema. This was adjusted to the indicators at the schema stage that subject 1 was expected to fully understand the concepts of cubes and cuboids. The item used to describe the scheme stage was found in item number 3. The subject could understand the relationship and comparison of two or more figures with different sizes. Based on the interview, subject 1 showed that there were other elements such as the diagonal space and diagonal plane which would also change when the side length of a shape changes. She also added that to determine the surface area if it changed twice, the ratio of the area would be 1:4. Subject 1 made a schema of knowledge from a series of understanding of the concept of cube and cuboid. Next, to understand the concept of the volume, the length, width, and height of cubes and cuboids must be known. Based on the explanation provided by subject 1, it can be concluded that she had combined several stages in the APOS framework systematically. 
- Subject 1 completed all mental constructions in the APOS framework. On the other hand, Ndlovu \& Brijlall (2016) found that students with a high interest in learning made a model of the mathematics problem. Finally, the student-organized all action, process, and object well to make schema. Also, the APOS framework could reduce students' mistakes to solve the description question of the cube and cuboid problems (Altieri \& Schirmer, 2019).

\subsection{The Research Result Based on Subject 2}

Subject 2 could solve the cube and cuboid task that was given in item number 3 . The results showed that she solved all the problems in the task well although some things needed to be confirmed through interviews.

\subsubsection{Action Stage}

At the action stage, subject 2 could explain the purpose of the problem. She also solved problems in item number 1 related to surface area and volume of cube and cuboid. Subject 2 determined the surface area and volume by incorporating known elements into the formula. The formula for surface area and volume of cube and cuboid was correct. Figure 4 shows the subject 2 work result on the problem that contains the indicators of the action stage.

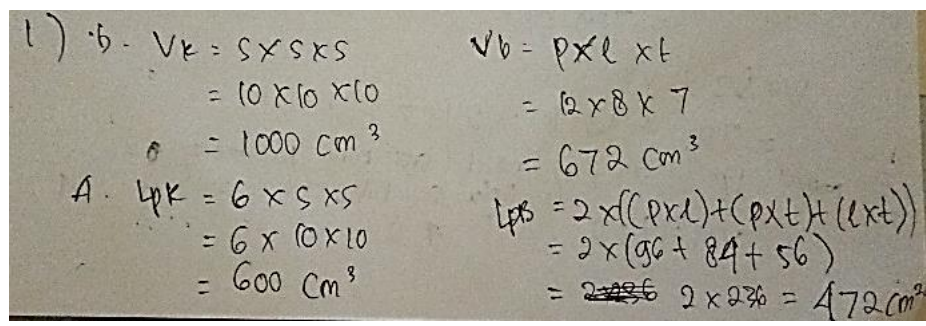

Figure 4. Subjects 2 Work Results in Item Number 1

The results showed that subject 2 could determine the surface area and volume of the cube and cuboid correctly. Thus, this stage can be carried out well by subject 2 .

\subsubsection{Process Stage}

The process stage contained some indicators where the subject was expected to explain the steps in solving the problem in item number 2. The results showed that subject 2 could understand the problem by showing the results and explain it. She also explained the steps in determining the desired volume in item number 2 but the subject had difficulty determining the surface area. The following is the result of item number 2 .

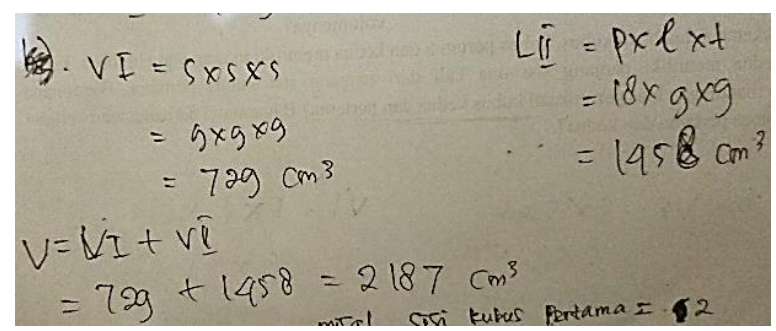

Figure 5. Subjects 2 Work Results in Item Number 2

Figure 5 shows that subject 2 determined the volume correctly. Besides, she could also explain the steps in determining the volume. So, subject 2 had carried out the process stage well even though there were some mistakes. 


\subsubsection{Object Stage}

Subject 2 could understand the problem in item number 3. She explained the purpose of the problem during the interview. The subject understood the comparison of two figures of different sizes. She made an example by drawing two cubes with a length of $2 \mathrm{~cm}$ in the first figure, then in the second figure, the size changes twice then the subject concluded that the length was $4 \mathrm{~cm}$ with a ratio of $1: 2$. She said that because the cube had the same side length, so it was easy to compare. On the other hand, when subject 2 was asked further related to the surface area and volume, she stated that if the ratio was $1: 2$, then the others should be the same. Thus, subject 2 had completed the criteria of the indicator of the object stage although some things should be maximized.

\subsubsection{Schema Stage}

The item used to describe the schema stage was found in item number 3 . The subject was expected to explain the relationship and comparison of two or more figures that had different sizes. The answers, confirmed through the interview, showed that subject 2 did not understand that the diagonal face and diagonal plane will change when the side length of a figure changes. Subject 2 answered that she did not understand the other elements. On the other hand, she only showed the diagonal face and diagonal space. For the diagonal plane, the subject could not answer the question. Also, when the researchers asked the method to determine the length of the diagonal face, he could not explain in detail. Based on this description, it can be concluded that subject 2 could not correlate the action, process, and object stages appropriately so that the schema stage was not optimum.

Vidakovic et al. (2018) explained that students with a medium interest in learning could only complete two APOS stages. Moreover, Anam et al. (2019) conclude that students who have medium mathematical ability could not solve the problem. Thus, the student did not fulfill all criteria of the schema stage. That result was similar to this research result in which subject 2 could only complete three stages of all stages in APOS.

\subsection{The research result based on subject 3}

Subject 3 could only answer question 1 . She made some errors, therefore, the researchers could not obtain complete data from subject 3 .

\subsubsection{Action Stage}

Item 1 contained indicators at the action stage that could be done well by subject 3 . The problem provided was not so complex. The subject was expected to determine the surface area and volume of cube and cuboid procedurally. The results of work on item 2 showed that subject 3 understood the problem by writing the formula for the surface area of cube and cuboid and wrote the volume formula. Next, she applied the formula to the elements known in the figure. The subject provided the correct answer. Thus, subject 3 has carried out the action stage properly according to the indicators contained in the task.

\subsubsection{Process Stage}

In this stage, subject 3 was less successful in implementing the indicators. She could only understand the problem without being able to show the workmanship and explained it. When confirmed through interviews, subject 3 suggested that the structure contained in item 2 was a pile of cube and cuboid of different sizes. Next, the researchers asked about the surface area and volume of the figures. The subject had difficulty and could not explain it. 


\subsubsection{Object and Schema Stages}

In item 3 which contained indicators of object and schema stages, subject 3 was not able to understand the problem well. She did not fully understand the elements contained in the cube and cuboid and not yet understood the concept of surface area and volume of cube and cuboid well. This fact was based on the results of interviews with the subject. Therefore, at the object and schema stage, subject 3 did not meet the criteria of the indicators. There were some unsuccessful responses of the students with the weak mathematical ability (Borji et al., 2018). They had some difficulties in prerequisite concepts for solving problems.

\section{CONCLUSIONS}

This research found that each of the subjects had different mathematical abilities and different mental construction. The mental constructions described through the stages of action, process, object, and schema had different explanations for each subject. In the action stage, all three subjects could complete the cube and cuboid tasks correctly. In the process stage, subjects with high mathematical ability provided detailed explanations of the steps to determine the surface area and the volume of cube and cuboid and also understood each step. On the other hand, the subject with medium mathematical ability explained the steps to determine the volume well but there was an error when the subject determined the surface area. Meanwhile, the subject with the low mathematical ability only showed the formula to determine the surface area and volume of cube and cuboid. The action and process stages were carried out and understood correctly, so it became a cognitive object. For the object stage, the subject with high mathematical ability could explain the comparisons of two different shapes and their sizes. Detailed comparisons of the surface area and volume of cube and cuboid could be determined by the subject perfectly. The subject with medium mathematical ability explained the comparison of two shapes with different sizes and determined their size alteration. The subject could explain the ratio of the surface area and volume of cube well but not with the cuboid. The subject with the low mathematical ability only showed the comparison of two shapes that had different sizes without being able to explain them. In the schema stage, subjects with high mathematical ability made a systematic understanding related to the concepts of surface area and volume of cube and cuboid. Moreover, the other two subjects could only explain their understanding separately without explaining the elements of the surface area and volume and they did not make detailed and systematic explanations.

It is recommended for teachers to apply the scaffolding to students who need improvement. For the next research, it is essential to investigate students' cognitive obstacles in concept understanding.

\section{AUTHOR CONTRIBUTIONS STATEMENT}

IR, ACA, and PES worked as the main drafter in this research. data collection and instrument design assisted by WHI and IS. Next, they create articles. 


\section{REFERENCES}

Altieri, M., \& Schirmer, E. (2019). Learning the concept of eigenvalues and eigenvectors: A comparative analysis of achieved concept construction in linear algebra using APOS theory among students from different educational backgrounds. ZDM, 51(7), 1125-1140.

Anam, A. C., Juniati, D., \& Wijayanti, P. (2019). Understanding the quadrilateral concept of junior high school students based on APOS theory in terms of differences in cognitive styles, Proceedings on Mathematics, Informatics, Science, and Education International Conference (MISEIC 2019). Atlantis Press.

Anwar, \& Rofiki, I. (2018). Investigating students' learning trajectory: A case on triangle. Journal of Physics: Conference Series, 1088(1), 012021.

Arnon, I., Cottrill, J., Dubinsky, E., Oktaç, A., Fuentes, S. R., Trigueros, M., \& Weller, K. (2014). The APOS paradigm for research and curriculum development. In APOS Theory (pp. 93-108). New York: Springer.

Bansilal, S., Deonarain B., \& Maria T. (2017). An APOS study on pre-service teachers' understanding of injections and surjections. Journal of Mathematical Behavior, 48, 2237.

Barmby, P., Harries T., Higgins S., \& Suggate. (2007). How can we assess mathematical understanding. Proceedings on the 31"of the International Group for the Psychology of Mathematics Education. 41-48. Seoul: PME.

Barody, A. J., Feil Y., \& Johson A. R. (2007). An alternative reconceptualization of procedural and conceptual knowledge. Journal for Research in Mathematics Education, 38, 115-131.

Borji, V., Alamolhodaei, H., \& Radmehr, F. (2018). Application of the APOS-ACE theory to improve students' graphical understanding of derivative. EURASIA Journal of Mathematics, Science and Technology Education, 14(7), 2947-2967.

Brijlall, D., \& Ndlovu, Z. (2013). High school learners' mental construction during solving optimisation problems in calculus: A South African case study. South African Journal of Education, 33(2), 1-18.

Dubinsky, E. (2002). Reflective abstraction in advanced mathematical thinking. In Advanced mathematical thinking (pp. 95-126). Springer, Dordrecht.

Dubinsky, E., \& Wilson, T. R. (2001). High school students' understanding of the function concept. Journal of Mathematical Behavior, 32(1), 83-101.

Figueroa, A. P., Possani, E., \& Trigueros, M. (2018). Matrix multiplication and transformations: An APOS approach. The Journal of Mathematical Behavior, 52, 77-91.

García, M., I., \& Parraguez, M. (2017). The basis step in the construction of the principle of mathematical induction based on APOS theory. The Journal of Mathematical Behavior, $46,128-143$. 
Gray, E., Pinto, M., Pitta, D., \& Tall, D. (1999). Knowledge construction and diverging thinking in elementary \& advanced mathematics. Educational studies in mathematics, 38(1-3), 111-133.

Haylock, D., \& Cockburn, A. D. (2008). Understanding mathematics for young children: A guide for foundation stage and lower primary teachers. United Kingdom: SAGE Publication.

Kurniawan, A. P., Anam, A. C., Abdussakir, \& Rofiki, I. (2019). Integrasi etnomatematika dengan model pembelajaran probing-prompting untuk melatih komunikasi matematis siswa. Mapan: Jurnal Matematika dan Pembelajaran, 7(1), 1-15.

Martínez-Planell, R., \& Delgado, A. C. (2016). The unit circle approach to the construction of the sine and cosine functions and their inverses: An application of APOS theory. The Journal of Mathematical Behavior, 43, 111-133.

Mena-Lorca, A., \& Parraguez, A. M. M. (2016). Mental constructions for the group isomorphism theorem. International Electronic Journal of Mathematics Education, 11(2), 377-393.

Ndlovu, Z., \& Brijlall, D. (2016). Pre-service mathematics teachers' mental constructions of the determinant concept. International Journal of Educational Sciences, 14(1-2), 145156.

Ndlovu, Z., \& Brijlall, D. (2019). Pre-service mathematics teachers' mental constructions when using Cramer's rule. South African Journal of Education, 39(1), 1-13.

Oktaç, A. (2019). Mental constructions in linear algebra. ZDM, 51(7), 1043-1054.

Priss, U. (2018). Combining and contrasting formal concept analysis and APOS theory. In International Conference on Conceptual Structures (pp. 96-104). Springer, Cham.

Puloo, M. M. L., Juniati, D., \& Wijayanti, P. (2018). Visualization profile of junior high school students in solving geometry problems viewed from gender differences. Journal of Physics: Conference Series, 1108(1), 012063.

Rofiki, I., Nusantara, T., Subanji, S., \& Chandra, T. D. (2017). Reflective plausible reasoning in solving inequality problem. IOSR Journal of Research \& Method in Education (IOSRJRME), 7(1), 101-112.

Suryani, A. I., Anwar, Hajidin, \& Rofiki, I. (2020). The practicality of mathematics learning module on triangles using GeoGebra. Journal of Physics: Conference Series, 1470(1), 012079.

Vidakovic, D., Dubinsky, E., \& Weller, K. (2018). APOS theory: Use of computer programs to foster mental constructions and student's creativity. In Creativity and Technology in 